\title{
Interpolative Prešić Type Contractions and Related Results
}

\author{
Monairah Alansari ${ }^{1}$ and Muhammad Usman Ali $\mathbb{D}^{2}$ \\ ${ }^{1}$ Department of Mathematics, King Abdulaziz University, P.O. Box 80203, Jeddah 21589, Saudi Arabia \\ ${ }^{2}$ Department of Mathematics, COMSATS University Islamabad, Attock Campus, Attock, Pakistan
}

Correspondence should be addressed to Muhammad Usman Ali; muh_usman_ali@yahoo.com

Received 19 November 2021; Accepted 16 January 2022; Published 21 February 2022

Academic Editor: Antonio Francisco Roldán López de Hierro

Copyright (C) 2022 Monairah Alansari and Muhammad Usman Ali. This is an open access article distributed under the Creative Commons Attribution License, which permits unrestricted use, distribution, and reproduction in any medium, provided the original work is properly cited.

\begin{abstract}
In this article, we will extend the notion of interpolative Kannan contraction by introducing the notions of interpolative Prešić type contractions and interpolative Prešić type proximal contractions for mappings defined on product spaces. Through these notions, we will derive some results to ensure the existence of fixed points and best proximity points for such mappings.
\end{abstract}

\section{Introduction and Preliminaries}

The Banach contraction principle is the most significant and basic result of metric fixed point theory. Through this result, we can obtain a unique fixed point of a self-map $N: L \longrightarrow L$, provided that $N$ is a contraction map on a complete metric space $\left(L, d_{L}\right)$. This result motivated Prešić to study about the existence of fixed points of the operators defined on product spaces, that is, $N: L^{k} \longrightarrow L$, for any fixed $k \in \mathbb{N}$. As an outcome of this motivation, Prešić [1] presented the following noteworthy extension of the Banach contraction principle.

Theorem 1 (see [1]). Suppose that $\left(L, d_{L}\right)$ be a complete metric space and $N: L^{k} \longrightarrow L$ be a map, for any fixed $k \in \mathbb{N}$, satisfying the following inequality:

$d\left(N\left(l_{1}, l_{2}, \ldots, l_{k}\right), N\left(l_{2}, l_{3}, \ldots, l_{k+1}\right)\right) \leq \sum_{j=1}^{k} \zeta_{j} d_{L}\left(l_{j}, l_{j+1}\right)$,

for every $l_{1}, l_{2}, \ldots, l_{k}, l_{k+1} \in L$, where $\zeta_{1}, \zeta_{2}, \ldots, \zeta_{k}$ are the nonnegative real numbers with $\sum_{j=1}^{k} \zeta_{j}<1$. Then, there exists a unique point of $L$ that satisfies the equation $l=N(\underbrace{l, l, \ldots, l}_{k})$.

This result is used to discuss the existence of equilibrium points for the $k^{\text {th }}$-order nonlinear difference equation of the form

$$
l_{n+k}=N\left(l_{n}, l_{n+1}, \ldots, l_{n+k-1}\right),
$$

where $N: L^{k} \subset \mathbb{R}^{k} \longrightarrow L$ is a continuous map. Note that a point $l^{*} \in L$ is known as an equilibrium point of (2) if $l^{*}=N(\underbrace{l^{*}, l^{*}, \ldots, l^{*}}_{k})$. Such a point is also known as a fixed point of $N: L^{k} \longrightarrow L$. Some well-known generalizations of this work have been studied by several authors, for example, [2-5].

Kannan and Chatterjea made a vital contribution in the development of this field through the fixed point results derived in [6, 7], respectively. Recently, Karapınar [8] modified the Kannan contraction by introducing interpolative Kannan contraction, stated as, a map $N:\left(L, d_{L}\right) \longrightarrow\left(L, d_{L}\right)$ is called an interpolative Kannan contraction [8] if

$$
d_{L}(N k, N l) \leq \zeta d_{L}(k, N k)^{\vartheta} d_{L}(l, N l)^{1-\vartheta},
$$

for all $k, l \in L$ with $k \neq N k$ and $l \neq N l$, where $\zeta \in[0,1)$ and $\vartheta \in(0,1)$.

After that, many existing contraction-type conditions have been generalized in the sense of interpolative Kannan contraction, for example, Karapınar et al. [9] studied interpolative Reich-Rus-Ćirić type contraction in partial metric spaces, Aydi et al. [10] studied interpolative ĆirićReich-Rus type contractions in Branciari metric spaces, Mohammadi et al. [11] extended the concept of F-contractions by interpolative Ćirić-Reich-Rus type 
F-contractions, Karapınar et al. [12] studied interpolative Hardy-Rogers type contractions, Debnath and Sen [13] studied set-valued interpolative Hardy-Rogers and set-valued Reich-Rus-Ćirić-type contractions, Sarwar et al. [14] presented rational type interpolative contractions, Khan et al. [15] worked on interpolative $(\phi, \psi)$-type $Z$-contractions, Altun and Tasdemir [16] presented interpolative proximal contractions for nonself mappings, Fulga and Yesilkaya [17] studied interpolative Suzuki-type contractions, Karapinar et al. [18] defined $(\alpha, \beta, \psi, \phi)$-interpolative contractions, and Alansari and Ali [19] studied multivalued interpolative Reich-Rus-Ćirić-type contractions.

Gaba and Karapinar [20] extended the notion of interpolative Kannan contraction through exponential powers, stated as, a map $N:\left(L, d_{L}\right) \longrightarrow\left(L, d_{L}\right)$ is called an $\left(\zeta, \vartheta_{1}, \vartheta_{2}\right)$-interpolative Kannan contraction, if

$$
d_{L}(N k, N l) \leq \zeta d_{L}(k, N k)^{\vartheta_{1}} d_{L}(l, N l)^{\vartheta_{2}},
$$

for all $k, l \in L$ with $k \neq N k$ and $l \neq N l$, where $\vartheta_{1}, \vartheta_{2} \in(0,1)$ with $\vartheta_{1}+\vartheta_{2}<1$ and $\zeta \in[0,1)$. Readers can find other similar generalizations in [21].

Consider $O_{L}$ and $M_{L}$ be nonvoid subsets of a metric space $\left(L, d_{L}\right)$. It is well-known that a fixed point of a map $N: O_{L} \longrightarrow M_{L}$ is a solution of $N l=l$. If $O_{L} \cap M_{L}=\varnothing$, then fixed point of $N: O_{L} \longrightarrow M_{L}$ does not exist, that is, $d_{L}(N l, l)>0$ for all $l \in O_{L}$. In this situation, we try to find $l \in O_{L}$, such that $d_{L}(N l, l)$ attain the minimum value in some sense. It is obvious that the smallest value that can be obtained by $d_{L}(N l, l)$ for any $l \in O_{L}$ will be greater or equal to $D_{L}\left(O_{L}, M_{L}\right)$, that is, distance between $O_{L}$ and $M_{L}$. A point $l \in O_{L}$ is said to be a best proximity point of $N: O_{L} \longrightarrow M_{L}$, if $d_{L}(N l, l)=D_{L}\left(O_{L}, M_{L}\right)$. The existence of such points of nonself maps has been discussed by several researchers in different ways, for example, Caballero et al. [22] studied the existence of best proximity points for nonself maps satisfying Geraghty contraction and $P$-property in metric spaces, Bilgili et al. [23], Aydi et al. [24], and Pitea [25] extended the work of Caballero et al. [22] by introducing generalized Geraghty contraction, $\psi$-Geraghty contraction and generalized almost $\theta$-Geraghty contraction for nonself maps, Basha and Shahzad [26] and Basha [27] defined proximal-type contractions to study the existence of best proximity points, Jleli and Samet [28] defined $\alpha$ - $\psi$-proximal contraction to ensure the existence of best proximity points, Jleli et al. [29] and Aydi et al. [30] defined generalized $\alpha$ - $\psi$-proximal contractions to extend the work of Jleli and Samet [28], Abkar and Gabeleh [31] and Kumam et al. [32] studied the existence of best proximity points for multivalued nonself maps in metric spaces, Ali et al. [33] defined implicit proximal contractions, Sahin et al. [34] defined proximal nonunique contraction, and Ali et al. [35] studied the existence of best proximity points for Prešić type nonself operators satisfying proximal type contractions.

This article aims to present the notions of interpolative Prešić type contractions and interpolative Prešić type proximal contractions for mappings defined on product spaces. Through these notions, we will study the existence of fixed points and best proximity points for such mappings.

\section{Main Results}

We begin this section with the following definition.

Definition 1. A map $N: L \times L \longrightarrow L$ is called an interpolative Prešić type-I contraction, if for each $s, w, t, v \in L \backslash F i x(N)$, we get

$$
d_{L}(N(s, w), N(t, v))^{\min \{\gamma(s, w), \gamma(t, v)\}} \leq \zeta d_{L}(w, N(s, w))^{\vartheta_{1}} d_{L}(v, N(t, v))^{\vartheta_{2}},
$$

where $\gamma: L \times L \longrightarrow \mathbb{R} \backslash\{0\}$ is a map, $\vartheta_{1}, \vartheta_{2} \in(0,1)$ with $\vartheta_{1}+\vartheta_{2}=1, \zeta \in[0,1)$, and Fix $(N)=\{l \in L: l=N(l, l)\}$.

The following theorem is used to study the existence of fixed points for the above map.

Theorem 2. Consider an interpolative Prešić type-I contraction map $N: L \times L \longrightarrow L$ on a complete metric space $\left(L, d_{L}\right)$. Also, consider that

(i) If $\min \{\gamma(s, w), \gamma(t, v)\}=1$, then $\gamma(N(s, w)$, $N(t, v))=1$.

(ii) There exist two elements $s, w \in L$ with $\min \{\gamma(s, w), \gamma(w, N(s, w))\}=1$.

(iii) For every sequence $\left\{l_{m}\right\}$ in $L$ with $\gamma\left(l_{m}, l_{m+1}\right)=1 \forall m \geq m_{0}$ for some natural number $m_{0}$ and $l_{m} \longrightarrow l$, we have $\gamma(l, l)=1$.
Then, there exists at least one point of $L$ that satisfies the equation $l=N(l, l)$.

Proof. Hypothesis (ii) assures that there are two points, say, $l_{0}$ and $l_{1}$ in $L$ with

$$
\min \left\{\gamma\left(l_{0}, l_{1}\right), \gamma\left(l_{1}, N\left(l_{0}, l_{1}\right)\right)\right\}=1 .
$$

By using these two points, we can define a sequence $\left\{l_{m}\right\}$ with $l_{m+1}=N\left(l_{m-1}, l_{m}\right) \forall m \in \mathbb{N}$. From hypothesis (i), it can be concluded that $\gamma\left(l_{m}, l_{m+1}\right)=1, \forall m>1$. Hence,

$$
\min \left\{\gamma\left(l_{m-1}, l_{m}\right), \gamma\left(l_{m}, l_{m+1}\right)\right\}=1 \forall m \in \mathbb{N} .
$$

By (5), we get 


$$
\begin{aligned}
d_{L}\left(N\left(l_{m-1}, l_{m}\right), N\left(l_{m}, l_{m+1}\right)\right) & =d_{L}\left(N\left(l_{m-1}, l_{m}\right), N\left(l_{m}, l_{m+1}\right)\right)^{\min \left\{\gamma\left(l_{m-1}, l_{m}\right), \gamma\left(l_{m}, l_{m+1}\right)\right\}}, \\
& \leq \zeta d_{L}\left(l_{m}, N\left(l_{m-1}, l_{m}\right)\right)^{\vartheta_{1}} d_{L}\left(l_{m+1}, N\left(l_{m}, l_{m+1}\right)\right)^{\vartheta_{2}} \forall m \in \mathbb{N}, \\
& d_{L}\left(l_{m+1}, l_{m+2}\right) \leq \zeta^{m} d_{L}\left(l_{1}, l_{2}\right), \quad \forall m \in \mathbb{N} .
\end{aligned}
$$

that is,

$d_{L}\left(l_{m+1}, l_{m+2}\right) \leq \zeta d_{L}\left(l_{m}, l_{m+1}\right)^{\vartheta_{1}} d_{L}\left(l_{m+1}, l_{m+2}\right)^{\vartheta_{2}}, \quad \forall m \in \mathbb{N}$.

By (9), we obtain

$d_{L}\left(l_{m+1}, l_{m+2}\right)^{1-\vartheta_{2}} \leq \zeta d_{L}\left(l_{m}, l_{m+1}\right)^{\vartheta_{1}}, \quad \forall m \in \mathbb{N}$.

Since $1-\vartheta_{2}=\vartheta_{1}$, thus, by $(10)$, we get

$$
\begin{aligned}
d_{L}\left(l_{m+1}, l_{m+2}\right) & \leq \zeta^{1 /\left(1-\vartheta_{2}\right)} d_{L}\left(l_{m}, l_{m+1}\right) \\
& \leq \zeta d_{L}\left(l_{m}, l_{m+1}\right) \forall m \in \mathbb{N}
\end{aligned}
$$

Hence, by (11), we conclude that
By triangle inequality and (12), for each $k, n \in \mathbb{N}$ with $k>n$, we obtain

$$
d_{L}\left(l_{n}, l_{k}\right) \leq \sum_{j=n}^{k-1} d_{L}\left(l_{j}, l_{j+1}\right) \leq \sum_{j=n}^{k-1} \zeta^{j-1} d_{L}\left(l_{1}, l_{2}\right) .
$$

In view of the above inequality and the convergence of $\sum_{j=1}^{\infty} \zeta^{j}$, we say that the sequence $\left\{l_{m}\right\}$ is a Cauchy in $L$. By the completeness of $\left(L, d_{L}\right)$, we get a point $l^{*} \in L$, such that $l_{m} \longrightarrow l^{*}$. Also, by (iii), we get $\gamma\left(l^{*}, l^{*}\right)=1$, since $\gamma\left(l_{m}, l_{m+1}\right)=1, \quad \forall m \in \mathbb{N}$ and $l_{m} \longrightarrow l^{*}$.

Here, the claim is $l^{*}=N\left(l^{*}, l^{*}\right)$. If the claim is wrong, then by (5), for each $m \in \mathbb{N}$, we get

$$
\begin{aligned}
d_{L}\left(N\left(l_{m-1}, l_{m}\right), N\left(l^{*}, l^{*}\right)\right) & =d_{L}\left(N\left(l_{m-1}, l_{m}\right), N\left(l^{*}, l^{*}\right)\right)^{\min \left\{\gamma\left(l_{m-1}, l_{m}\right), \gamma\left(l^{*}, l^{*}\right)\right\}}, \\
& \leq \zeta d_{L}\left(l_{m}, N\left(l_{m-1}, l_{m}\right)\right)^{\vartheta_{1}} d_{L}\left(l^{*}, N\left(l^{*}, l^{*}\right)\right)^{\vartheta_{2}}, \\
& \leq \zeta d_{L}\left(l_{m+1}, l_{m+2}\right){ }^{\vartheta_{1}} d_{L}\left(l^{*}, N\left(l^{*}, l^{*}\right)\right)^{\vartheta_{2}} .
\end{aligned}
$$

By triangle inequality and (14), we obtain

$$
\begin{aligned}
d_{L}\left(l^{*}, N\left(l^{*}, l^{*}\right)\right) & \leq d_{L}\left(l^{*}, N\left(l_{m}, l_{m+1}\right)\right)+d_{L}\left(N\left(l_{m}, l_{m+1}\right), N\left(l^{*}, l^{*}\right)\right), \\
& \leq d_{L}\left(l^{*}, l_{m+2}\right)+\zeta d_{L}\left(l_{m+1}, l_{m+2}\right)^{\vartheta_{1}} d_{L}\left(l^{*}, N\left(l^{*}, l^{*}\right)\right)^{\vartheta_{2}} .
\end{aligned}
$$

Letting $m \longrightarrow \infty$ in (15), we get $d_{L}\left(l^{*}, N\left(l^{*}, l^{*}\right)\right)=0$. Hence, the claim is true, that is, $l^{*}=N\left(l^{*}, l^{*}\right)$.

Example 1. Consider $L=\mathbb{R}$ equipped with a metric $d_{L}(k, l)=|k-l|$ for each $k, l \in L$. Define $N: L \times L \longrightarrow L$ and $\gamma: L \times L \longrightarrow \mathbb{R} \backslash\{0\}$ by

$$
N(k, l)= \begin{cases}\frac{k+l}{2}, & \text { if } k, l \geq 0, \\ 0, & \text { otherwise, }\end{cases}
$$

and

$$
\gamma(k, l)= \begin{cases}1, & \text { if } k, l \geq 0 . \\ 1 / 2, & \text { otherwise. }\end{cases}
$$

Then, one can easily verify that the axioms of Theorem 2 are satisfied. Hence, there is at least one element $l^{*} \in L$, such that $l^{*}=N\left(l^{*}, l^{*}\right)$.

In the following, we present interpolative Prešić type-II contraction map and related fixed point result.
Definition 2. A map $N: L \times L \longrightarrow L$ is called an interpolative Prešić type-II contraction, if for each $s, w, t, v \in L \backslash \operatorname{Fix}(N)$ with $\min \{\gamma(s, w), \gamma(t, v)\} \geq 1$, we get $d_{L}(N(s, w), N(t, v)) \leq \zeta d_{L}(w, N(s, w))^{\vartheta_{1}} d_{L}(v, N(t, v))^{\vartheta_{2}}$,

where $\gamma: L \times L \longrightarrow \mathbb{R}$ is a map, $\vartheta_{1}, \vartheta_{2} \in(0,1)$ with $\vartheta_{1}+\vartheta_{2}=1, \zeta \in[0,1)$, and $\operatorname{Fix}(N)=\{l \in L: l=N(l, l)\}$.

Theorem 3. Consider an interpolative Prešić type-II contraction map $N: L \times L \longrightarrow L$ on a complete metric space $\left(L, d_{L}\right)$. Also, consider that

(i) If $\min \{\gamma(s, w), \gamma(t, v)\} \geq 1$, then $\gamma(N(s, w)$, $N(t, v)) \geq 1$.

(ii) There exist two elements $s, w \in L \quad$ with $\min \{\gamma(s, w), \gamma(w, N(s, w))\} \geq 1$.

(iii) For every sequence $\left\{l_{m}\right\}$ in $L$ with $\gamma\left(l_{m}, l_{m+1}\right) \geq 1 \forall m \geq m_{0}$ for some natural number $m_{0}$ and $l_{m} \longrightarrow l$, we have $\gamma(l, l) \geq 1$. 
Then, there exists at least one point of $L$ that satisfies the equation $l=N(l, l)$.

Proof. In view of the hypothesis (ii), we get

$$
\min \left\{\gamma\left(l_{0}, l_{1}\right), \gamma\left(l_{1}, N\left(l_{0}, l_{1}\right)\right)\right\} \geq 1 .
$$

For some $l_{0}$ and $l_{1}$ in $L$. Through these two points, we can construct a sequence $\left\{l_{m}\right\}$ with $l_{m+1}=N\left(l_{m-1}, l_{m}\right) \forall m \in \mathbb{N}$. Also, hypothesis (i) implies that $\gamma\left(l_{m}, l_{m+1}\right) \geq 1, \quad \forall m>1$. Hence,

$$
\min \left\{\gamma\left(l_{m-1}, l_{m}\right), \gamma\left(l_{m}, l_{m+1}\right)\right\} \geq 1 \forall m \in \mathbb{N} .
$$

By (18), we get

$$
d_{L}\left(N\left(l_{m-1}, l_{m}\right), N\left(l_{m}, l_{m+1}\right)\right) \leq \zeta d_{L}\left(l_{m}, N\left(l_{m-1}, l_{m}\right)\right)^{\vartheta_{1}} d_{L}\left(l_{m+1}, N\left(l_{m}, l_{m+1}\right)\right)^{\vartheta_{2}} \forall m \in \mathbb{N} .
$$

By performing some calculations, we get

$$
d_{L}\left(l_{m+1}, l_{m+2}\right) \leq \zeta d_{L}\left(l_{m}, l_{m+1}\right)^{\vartheta_{1}} \leq \zeta^{m} d_{L}\left(l_{1}, l_{2}\right), \quad \forall m \in \mathbb{N} .
$$

Hence, it can be seen that $\left\{l_{m}\right\}$ is a Cauchy sequence in $L$ with $l_{m} \longrightarrow l^{*} \in L$. Also, by (iii), we get $\gamma\left(l^{*}, l^{*}\right) \geq 1$. Suppose that $l^{*} \neq N\left(l^{*}, l^{*}\right)$. Then, by (18), for each $m \in \mathbb{N}$, we get

$$
\begin{aligned}
d_{L}\left(N\left(l_{m}, l_{m+1}\right), N\left(l^{*}, l^{*}\right)\right) & \leq \zeta d_{L}\left(l_{m+1}, N\left(l_{m}, l_{m+1}\right)\right)^{\vartheta_{1}} d_{L}\left(l^{*}, N\left(l^{*}, l^{*}\right)\right)^{\vartheta_{2}} \\
& \leq \zeta d_{L}\left(l_{m+1}, l_{m+2}\right)^{\vartheta_{1}} d_{L}\left(l^{*}, N\left(l^{*}, l^{*}\right)\right)^{\vartheta_{2}} .
\end{aligned}
$$

By triangle inequality and (23), we obtain

$$
\begin{aligned}
d_{L}\left(l^{*}, N\left(l^{*}, l^{*}\right)\right) & \leq d_{L}\left(l^{*}, N\left(l_{m}, l_{m+1}\right)\right)+d_{L}\left(N\left(l_{m}, l_{m+1}\right), N\left(l^{*}, l^{*}\right)\right) \\
& \leq d_{L}\left(l^{*}, l_{m+2}\right)+\zeta d_{L}\left(l_{m+1}, l_{m+2}\right)^{\vartheta_{1}} d_{L}\left(l^{*}, N\left(l^{*}, l^{*}\right)\right)^{\vartheta_{2}}
\end{aligned}
$$

Letting $m \longrightarrow \infty$ in (24), we get $d_{L}\left(l^{*}, N\left(l^{*}, l^{*}\right)\right)=0$. Hence, our supposition is wrong and $l^{*}=N\left(l^{*}, l^{*}\right)$.

Example 2. Consider $L=\mathbb{Z}$ equipped with a metric $d_{L}(k, l)=|k-l|$ for each $k, l \in L$. Define $N: L \times L \longrightarrow L$ and $\gamma: L \times L \longrightarrow \mathbb{R}$ by

$$
N(k, l)= \begin{cases}l, & \text { if } k, l \geq 0, \\ |k|+|l| & \text { otherwise, }\end{cases}
$$

and

$$
\gamma(k, l)= \begin{cases}1, & \text { if } k, l \geq 0 . \\ 0, & \text { otherwise. }\end{cases}
$$

Then, one can easily verify that the axioms of Theorem 3 are satisfied. Hence, there is at least one element $l^{*} \in L$, such that $l^{*}=N\left(l^{*}, l^{*}\right)$.

Some consequences of the above results can be obtained in the form of the following listed corollaries. The following corollary is obtained from Theorem 2 by considering $t=w$.

Corollary 1. Consider a map $N: L \times L \longrightarrow$ Lon a complete metric space $\left(L, d_{L}\right)$, such that for each $s, w, v \in L \backslash F i x(N)$, we get

$$
d_{L}(N(s, w), N(w, v))^{\min \{\gamma(s, w), \gamma(w, v)\}} \leq \zeta d_{L}(w, N(s, w))^{\vartheta_{1}} d_{L}(v, N(w, v))^{\vartheta_{2}}
$$


where $\gamma: L \times L \longrightarrow \mathbb{R} \backslash\{0\}$ is a map, $\vartheta_{1}, \vartheta_{2} \in(0,1)$ with $\vartheta_{1}+\vartheta_{2}=1$, and $\zeta \in[0,1)$. Also, consider that

(i) If $\min \{\gamma(s, w), \gamma(w, v)\}=1$, then $\gamma(N(s, w)$, $N(w, v))=1$

(ii) There exist two elements $s, w \in L \quad$ with $\min \{\gamma(s, w), \gamma(w, N(s, w))\}=1$

(iii) For every sequence $\left\{l_{m}\right\}$ in $L$ with $\gamma\left(l_{m}, l_{m+1}\right)=1 \forall m \geq m_{0}$ for some natural number $m_{0}$ and $l_{m} \longrightarrow l$, we have $\gamma(l, l)=1$

Then, there exists at least one point of $L$ that satisfies the equation $l=N(l, l)$.

The following corollary is a special case of Theorem 3 which can be obtained by considering $t=w$.

Corollary 2. Consider a map $N: L \times L \longrightarrow L$ on a complete metric space $\left(L, d_{L}\right)$, such that for each $s, w, v \in L \backslash F i x(N)$ with $\min \{\gamma(s, w), \gamma(w, v)\} \geq 1$, we get

$d_{L}(N(s, w), N(w, v)) \leq \zeta d_{L}(w, N(s, w))^{\vartheta_{1}} d_{L}(v, N(w, v))^{\vartheta_{2}}$,

where $\gamma: L \times L \longrightarrow \mathbb{R}$ is a map, $\vartheta_{1}, \vartheta_{2} \in(0,1)$ with $\vartheta_{1}+\vartheta_{2}=$ 1 and $\zeta \in[0,1)$. Also, consider that

(i) If $\min \{\gamma(s, w), \gamma(w, v)\} \geq 1$, $\gamma(N(s, w), N(w, v)) \geq 1$

(ii) There exist two elements $s, w \in L$ with $\min \{\gamma(s, w), \gamma(w, N(s, w))\} \geq 1$

(iii) For every sequence $\left\{l_{m}\right\}$ in $L$ with $\gamma\left(l_{m}, l_{m+1}\right) \geq 1 \forall m \geq m_{0}$ for some natural number $m_{0}$ and $l_{m} \longrightarrow l$, we have $\gamma(l, l) \geq 1$
Then, there exists at least one point of $L$ that satisfies the equation $l=N(l, l)$.

By defining $\gamma(s, w)=1$ for each $s, w \in L$ in Theorem 2 or Theorem 3, we get the following.

Corollary 3. Consider a map $N: L \times L \longrightarrow L$ on a complete metric space $\left(L, d_{L}\right)$ that satisfies

$d_{L}(N(s, w), N(t, v)) \leq \zeta d_{L}(w, N(s, w))^{\vartheta_{1}} d_{L}(v, N(t, v))^{\vartheta_{2}}$,

for each $s, w, v \in L \backslash$ Fix $(N)$, where $\vartheta_{1}, \vartheta_{2} \in(0,1)$ with $\vartheta_{1}+$ $\vartheta_{2}=1$ and $\zeta \in[0,1)$. Then, there exists at least one point of $L$ that satisfies the equation $l=N(l, l)$.

From the above corollary, we can also obtain the following result.

Corollary 4. Consider a map $N: L \times L \longrightarrow L$ on a complete metric space $\left(L, d_{L}\right)$ that satisfies

$d_{L}(N(s, w), N(w, v)) \leq \zeta d_{L}(w, N(s, w))^{\vartheta_{1}} d_{L}(v, N(w, v))^{\vartheta_{2}}$,

for each $s, w, v \in L \backslash F i x(N)$, where $\vartheta_{1}, \vartheta_{2} \in(0,1)$ with $\vartheta_{1}+$ $\vartheta_{2}=1$ and $\zeta \in[0,1)$. Then, there exists at least one point of $L$ that satisfies the equation $l=N(l, l)$.

In the following, we will study about the interpolative Prešić type proximal contractions and related results.

Let $\left(L, d_{L}\right)$ be a metric space and $O_{L}, M_{L}$ be nonvoid subsets of $L$. We will use the following notations.

$$
\begin{aligned}
D_{L}\left(O_{L}, M_{L}\right) & =\inf \left\{d_{L}(o, m): o \in O_{L}, m \in M_{L}\right\}, \\
d_{L}\left(o, M_{L}\right) & =\inf \left\{d_{L}(o, m): m \in M_{L}\right\}, \\
O_{L 0} & =\left\{o \in O_{L}: d_{L}(o, m)=D_{L}\left(O_{L}, M_{L}\right) \text { for some } m \in M_{L}\right\}, \\
M_{L 0} & =\left\{m \in M_{L}: d_{L}(o, m)=D_{L}\left(O_{L}, M_{L}\right) \text { for some } o \in O_{L}\right\} .
\end{aligned}
$$

Note that a point $o^{*} \in O_{L}$ is known as a best proximity point of $N: O_{L} \times O_{L} \longrightarrow M_{L}$ if $d_{L}\left(o^{*}\right.$, $\left.N\left(o^{*}, o^{*}\right)\right)=D_{L}\left(O_{L}, M_{L}\right)$. The collection of all such points for $N: O_{L} \times O_{L} \longrightarrow M_{L}$ is denoted by $\operatorname{Bes}(N)$.

Definition 3. A map $N: O_{L} \times O_{L} \longrightarrow M_{L}$ is called an interpolative Prešić type-I proximal contraction, if for each $s, w, t, v, p, q \in O_{L} \backslash \operatorname{Bes}(N)$ $d_{L}(p, N(s, w))=D_{L}\left(O_{L}, M_{L}\right)=d_{L}(q, N(t, v))$, we get

$$
d_{L}(p, q)^{\min \{\gamma(s, w), \gamma(t, v)\}} \leq \zeta d_{L}(w, p)^{\vartheta_{1}} d_{L}(v, q)^{\vartheta_{2}},
$$

where $\gamma: O_{L} \times O_{L} \longrightarrow \mathbb{R} \backslash\{0\}$ is a map, $\vartheta_{1}, \vartheta_{2} \in(0,1)$ with $\vartheta_{1}+\vartheta_{2}=1$ and $\zeta \in[0,1)$.

The following theorem is used to ensure the existence of best proximity points for the above defined maps.
Theorem 4. Consider an interpolative Prešić type-I proximal contraction map $N: O_{L} \times O_{L} \longrightarrow M_{L}$ on a metric space $\left(L, d_{L}\right)$. Also, consider that

(i) If $\quad \min \{\gamma(s, w), \gamma(t, v)\}=1 \quad$ and $d_{L}(p, N(s, w))=D_{L}\left(O_{L}, M_{L}\right)=d_{L}(q, N(t, v))$, then $\gamma(p, q)=1$.

(ii) There exist elements $s, w, p \in O_{L} \quad$ with $d_{L}(p, N(s, w))=D_{L}\left(O_{L}, M_{L}\right) \quad$ and $\min \{\gamma(s, w), \gamma(w, p)\} \geq 1$.

(iii) $N\left(O_{L} \times O_{L 0}\right) \subseteq M_{L 0}$.

(iv) $O_{L 0}$ is nonempty and complete with respect to $d_{L}$.

(v) For every sequence $\left\{l_{m}\right\}$ in $O_{L 0}$ with $\gamma\left(l_{m}, l_{m+1}\right)=$ $1 \forall m \geq m_{0}$ for some natural number $m_{0}$ and $l_{m} \longrightarrow l$, we have $\gamma(l, l)=1$. 
Then, there exists at least one point of L that satisfies the equation $d_{L}(l, N(l, l))=D_{L}\left(O_{L}, M_{L}\right)$.

Proof. From hypothesis (ii), we have $l_{0}, l_{1}$, and $l_{2}$ in $O_{L}$ with $\min \left\{\gamma\left(l_{0}, l_{1}\right) \gamma\left(l_{1}, l_{2}\right)\right\}=1$ and $d_{L}\left(l_{2}, N\left(l_{0}, l_{1}\right)\right)=D_{L}\left(O_{L}\right.$, $\left.M_{L}\right)$. Hypothesis (iii) implies that $N\left(l_{1}, l_{2}\right) \in M_{L_{0}}$, and there is $l_{3} \in O_{L 0}$ satisfying $d_{L}\left(l_{3}, N\left(l_{1}, l_{2}\right)\right)=D_{L}\left(O_{L}, M_{L}\right)$. Thus, from hypothesis (i), we get $\gamma\left(l_{2}, l_{3}\right)=1$. Hence, by using hypotheses (i) and (ii) repeatedly, we conclude that $\gamma\left(l_{m-1}, l_{m}\right)=1$ and $d_{L}\left(l_{m+1}, N\left(l_{m-1}, l_{m}\right)\right)=D_{L}\left(O_{L}, M_{L}\right)$ for all $m \in \mathbb{N}$.

Since
$\left(l_{m+2}, N\left(l_{m}, l_{m+1}\right)\right) \quad$ for $d_{L}\left(l_{m+1}, N\left(l_{m-1}, l_{m}\right)\right)=D_{L}\left(O_{L}, M_{L}\right)=$
each
$m \in \mathbb{N}$ $\min \left\{\gamma\left(l_{m-1}, l_{m}\right), \gamma\left(l_{m}, l_{m+1}\right)\right\}=1$ for each $m \in \mathbb{N}$, then, by (32), we get

$$
\begin{array}{r}
d_{L}\left(l_{m+1}, l_{m+2}\right) \leq \zeta d_{L}\left(l_{m+1}, l_{m+2}\right)^{\min \left\{\gamma\left(l_{m-1}, l_{m}\right), \gamma\left(l_{m}, l_{m+1}\right)\right\}}, \\
\leq \zeta d_{L}\left(l_{m}, l_{m+1}\right)^{\vartheta_{1}} d_{L}\left(l_{m+1}, l_{m+2}\right)^{\vartheta_{2}}, \quad \forall m \in \mathbb{N} .
\end{array}
$$

Now, by following the proof of Theorem 2, we say that $\left\{l_{m}\right\}_{m \geq 2}$ is a Cauchy sequence in $O_{L 0}$. Since $O_{L 0}$ is complete, we have a point $l^{*} \in O_{L 0}$, such that $l_{m} \longrightarrow l^{*}$. Also, by $(\mathrm{v})$, we get $\gamma\left(l^{*}, l^{*}\right)=1$, since $\gamma\left(l_{m}, l_{m+1}\right)=1$ and $l_{m} \longrightarrow l^{*}$. Clearly, $N\left(l^{*}, l^{*}\right) \in M_{L 0}$, and there is $w^{*} \in O_{L 0}$ with $d_{L}\left(w^{*}, N\left(l^{*}, l^{*}\right)\right)=D_{L}\left(O_{L}, M_{L}\right)$. Here, the claim is $w^{*}=l^{*}$. Suppose it is wrong, then by (33), for each $m \in \mathbb{N}$, we get

$$
\begin{aligned}
d_{L}\left(l_{m+1}, w^{*}\right) & =d_{L}\left(l_{m+1}, w^{*}\right)^{\min \left\{\gamma\left(l_{m-1}, l_{m}\right), \gamma\left(l^{*}, l^{*}\right)\right\}}, \\
& \leq \zeta d_{L}\left(l_{m}, l_{m+1}\right)^{\vartheta_{1}} d_{L}\left(l^{*}, w^{*}\right)^{\vartheta_{2}} .
\end{aligned}
$$

Letting $m \longrightarrow \infty$ in (34), we obtain $d_{L}\left(l^{*}, w^{*}\right)=0$, and it contradicts our assumption. Hence, our claim is true, that is, $l^{*}=w^{*}$. Therefore, $d_{L}\left(l^{*}, N\left(l^{*}, l^{*}\right)\right)=D_{L}\left(O_{L}, M_{L}\right)$.

In the following, we present the notion of interpolative Prešić type-II proximal contraction.

Definition 4. A map $N: O_{L} \times O_{L} \longrightarrow M_{L}$ is called an interpolative Prešić type-II proximal contraction, if for each $s, w, t, v, p, q \in O_{L} \backslash \operatorname{Bes}(N) \quad$ with $\quad d_{L}(p, N(s, w))=D_{L}$ $\left(O_{L}, M_{L}\right)=d_{L}(q, N(t, v))$ and $\min \{\gamma(s, w), \gamma(t, v)\} \geq 1$, we get

$$
d_{L}(p, q) \leq \zeta d_{L}(w, p)^{\vartheta_{1}} d_{L}(v, q)^{\vartheta_{2}}
$$

where $\gamma: O_{L} \times O_{L} \longrightarrow \mathbb{R}$ is a map, $\vartheta_{1}, \vartheta_{2} \in(0,1)$ with $\vartheta_{1}+$ $\vartheta_{2}=1$ and $\zeta \in[0,1)$.

The existence of best proximity points for above map can be ensured by the result given.

Theorem 5. Consider an interpolative Prešić type-II proximal contraction map $\mathrm{N}: \mathrm{O}_{L} \times \mathrm{O}_{L} \longrightarrow M_{L}$ on a metric space $\left(L, d_{L}\right)$. Also, consider that

(i) If $\min \{\gamma(s, w), \gamma(t, v)\} \geq 1$ and $d_{L}(p, N(s, w))=$ $D_{L}\left(O_{L}, M_{L}\right)=d_{L}(q, N(t, v))$, then $\gamma(p, q) \geq 1$.

(ii) There exist elements $s, w, p \in O_{L} \quad$ with $d_{L}(p, N(s, w))=D_{L}\left(O_{L}, M_{L}\right)$ and $\min \{\gamma(s, w)$, $\gamma(w, p)\} \geq 1$.

(iii) $N\left(O_{L} \times O_{L 0}\right) \subseteq M_{L 0}$.

(iv) $O_{L 0}$ is nonempty and complete with respect to $d_{L}$.

(v) For every sequence $\left\{l_{m}\right\}$ in $O_{L 0}$ with $\gamma\left(l_{m}, l_{m+1}\right)$ $\geq 1 \forall m \geq m_{0}$ for some natural number $m_{0}$ and $l_{m} \longrightarrow l$, we have $\gamma(l, l) \geq 1$.

Then, there exists at least one point of $L$ that satisfies the equation $d_{L}(l, N(l, l))=D_{L}\left(O_{L}, M_{L}\right)$.

Proof. The proof can be derived on the same steps as the proof of Theorem 4 is done.

2.1. Results for Extended Interpolative Prešić Type Maps. This subsection presents the extensions of the above listed results. Theorems 6 and 7 can be considered as an extended version of Theorem 2 and Theorem 3, respectively.

Theorem 6. Consider an extended interpolative Prešić type-I contraction map $N: L^{k} \longrightarrow L$, for any fixed $k \in \mathbb{N}$, on a complete metric space $\left(L, d_{L}\right)$, that is, for each $s_{1}, s_{2}, \ldots, s_{k}$, $w_{1}, w_{2}, \ldots, w_{k} \in L \backslash$ Fix $(N)$, we get

$$
\begin{aligned}
& d_{L}\left(N\left(s_{1}, s_{2}, \ldots, s_{k}\right), N\left(w_{1}, w_{2}, \ldots, w_{k}\right)\right)^{\min \left\{\gamma\left(s_{1}, w_{1}\right), \gamma\left(s_{2}, w_{2}\right), \ldots, \gamma\left(s_{k}, w_{k}\right)\right\}} \\
& \quad \leq \zeta d_{L}\left(s_{k}, N\left(s_{1}, s_{2}, \ldots, s_{k}\right)\right)^{\vartheta_{1}} d_{L}\left(w_{k}, N\left(w_{1}, w_{2}, \ldots, w_{k}\right)\right)^{\vartheta_{2}}
\end{aligned}
$$

where $\gamma: L \times L \longrightarrow \mathbb{R} \backslash\{0\}$ is a map, $\vartheta_{1}, \vartheta_{2} \in(0,1)$ with $\vartheta_{1}+\vartheta_{2}=1, \quad \zeta \in[0,1), \quad$ and $\operatorname{Fix}(N)=\{l \in L: l=N(l, l$, $\ldots, l)\}$. Also, consider that

(i) If $\min \left\{\gamma\left(s_{1}, w_{1}\right), \gamma\left(s_{2}, w_{2}\right), \ldots, \gamma\left(s_{k}, w_{k}\right)\right\}=1$, then $\gamma\left(N\left(s_{1}, s_{2}, \ldots, s_{k}\right), N\left(w_{1}, w_{2}, \ldots, w_{k}\right)\right)=1$.

(ii) There exist elements $s_{1}, s_{2}, \ldots, s_{k} \in L$, such that

$$
\min \left\{\gamma\left(s_{1}, s_{2}\right), \gamma\left(s_{2}, s_{3}\right), \ldots, \gamma\left(s_{k}, N\left(s_{1}, s_{2}, \ldots, s_{k}\right)\right)\right\}=1 .
$$

(iii) For every sequence $\left\{l_{m}\right\}$ in $L$ with $\gamma\left(l_{m}, l_{m+1}\right)=1 \forall m \geq m_{0}$ for some natural number $m_{0}$ and $l_{m} \longrightarrow l$, we have $\gamma\left(l_{m}, l\right)=1 \forall m \geq m_{0}$.

Then, there exists at least one point of $L$ that satisfies the equation $l=N(\underbrace{l, l, \ldots, l}_{k \text {-times }})$. 
Proof. Hypothesis (ii) implies the existence of elements $l_{1}, l_{2}, \ldots, l_{k}$ in $L$ with

$$
\min \left\{\gamma\left(l_{1}, l_{2}\right), \gamma\left(l_{2}, l_{3}\right), \ldots, \gamma\left(l_{k}, N\left(l_{1}, l_{2}, \ldots, l_{k}\right)\right)\right\}=1 .
$$

Through these points, we can define a sequence $\left\{l_{m}\right\}$ with $l_{m+k}=N\left(l_{m}, l_{m+1}, \ldots, l_{m+k-1}\right)$ for all $m \in \mathbb{N}$. Hence, by considering hypothesis (i), it can be concluded that $\gamma\left(l_{m}, l_{m+1}\right)=1 \forall m \geq m_{0}$. Then, we say that

$$
\min \left\{\gamma\left(l_{m}, l_{m+1}\right), \gamma\left(l_{m}, l_{m+1}\right), \ldots, \gamma\left(l_{m+k-1}, l_{m+k}\right)\right\}=1 \forall m \in \mathbb{N} .
$$

By (36), we get

$$
\begin{aligned}
d_{L}( & \left.N\left(l_{m}, l_{m+1}, \ldots, l_{m+k-1}\right), N\left(l_{m+1}, l_{m+2}, \ldots, l_{m+k}\right)\right) \\
= & d_{L}\left(N\left(l_{m}, l_{m+1}, \ldots, l_{m+k-1}\right), N\left(l_{m+1}, l_{m+2}, \ldots, l_{m+k}\right)\right)^{\min \left\{\gamma\left(l_{m}, l_{m+1}\right), \gamma\left(l_{m+1}, l_{m+2}\right), \ldots, \gamma\left(l_{m+k-1}, l_{m+k}\right)\right\}} \\
& \leq \zeta d_{L}\left(l_{m+k-1}, N\left(l_{m}, l_{m+1}, \ldots, l_{m+k-1}\right)\right)^{\vartheta_{1}} d_{L}\left(l_{m+k}, N\left(l_{m+1}, l_{m+2}, \ldots, l_{m+k}\right)\right)^{\vartheta_{2}} \forall m \in \mathbb{N}
\end{aligned}
$$

that is,

$$
d_{L}\left(l_{m+k}, l_{m+k+1}\right) \leq \zeta d_{L}\left(l_{m+k-1}, l_{m+k}\right)^{\vartheta_{1}} d_{L}\left(l_{m+k}, l_{m+k+1}\right)^{\vartheta_{2}}, \quad \forall m \in \mathbb{N}
$$

By (41), we obtain

$$
d_{L}\left(l_{m+k}, l_{m+k+1}\right)^{1-\vartheta_{2}} \leq \zeta d_{L}\left(l_{m+k-1}, l_{m+k}\right)^{\vartheta_{1}}, \quad \forall m \in \mathbb{N} \text {. }
$$

Since $1-\vartheta_{2}=\vartheta_{1}$, thus, by (42), we get

$$
\begin{aligned}
& d_{L}\left(l_{m+k}, l_{m+k+1}\right) \leq \zeta^{1 /\left(1-\vartheta_{2}\right)} d_{L}\left(l_{m+k-1}, l_{m+k}\right)^{\vartheta_{1}}, \\
& \leq \zeta d_{L}\left(l_{m+k-1}, l_{m+k}\right) \quad \forall m \in \mathbb{N} .
\end{aligned}
$$

Hence, by (43), we get

$$
d_{L}\left(l_{m+k}, l_{m+k+1}\right) \leq \zeta^{m} d_{L}\left(l_{k}, l_{k+1}\right), \quad \forall m \in \mathbb{N},
$$

that is,

$$
d_{L}\left(l_{m}, l_{m+1}\right) \leq \zeta^{m-k} d_{L}\left(l_{k}, l_{k+1}\right), \quad \forall m \geq k+1 .
$$

From triangle inequality and (45), for each $q, n \in \mathbb{N}$ with $q>n \geq k+1$, we obtain

$$
d_{L}\left(l_{n}, l_{q}\right) \leq \sum_{j=n}^{q-1} d_{L}\left(l_{j}, l_{j+1}\right) \leq \sum_{j=n}^{q-1} \zeta^{j-k} d_{L}\left(l_{k}, l_{k+1}\right) .
$$

Above inequality yields that $\left\{l_{m}\right\}$ is a Cauchy sequence in a complete space $L$. Hence, we get a point $l^{*} \in L$ with $l_{m} \longrightarrow l^{*}$. Also, by (iii), we get $\gamma\left(l_{m}, l^{*}\right)=1, \quad \forall m \in \mathbb{N}$, since $\gamma\left(l_{m}, l_{m+1}\right)=1, \quad \forall m \in \mathbb{N}$ and $l_{m} \longrightarrow l^{*}$.

Here, the claim is $l^{*}=N\left(l^{*}, l^{*}, \ldots, l^{*}\right)$. If the claim is wrong, then by (36), for each $m \in \mathbb{N}$, we get

$$
\begin{aligned}
d_{L}\left(N\left(l_{m}, l_{m+1}, \ldots, l_{m+k-1}\right), N\left(l^{*}, l^{*}, \ldots, l^{*}\right)\right) & =d_{L}\left(N\left(l_{m}, l_{m+1}, \ldots, l_{m+k-1}\right), N\left(l^{*}, l^{*}, \ldots, l^{*}\right)\right)^{\min \left\{\gamma\left(l_{m}, l^{*}\right), \gamma\left(l_{m+1}, l^{*}\right), \ldots, \gamma\left(l_{m+k-1}, l^{*}\right)\right\}} \\
& \leq \zeta d_{L}\left(l_{m+k-1}, N\left(l_{m}, l_{m+1}, \ldots, l_{m+k-1}\right)\right)^{\vartheta_{1}} d_{L}\left(l^{*}, N\left(l^{*}, l^{*}, \ldots, l^{*}\right)\right)^{\vartheta_{2}}, \\
& \leq d_{L}\left(l_{m+k-1}, l_{m+k}\right)^{\vartheta_{1}} d_{L}\left(l^{*}, N\left(l^{*}, l^{*}, \ldots, l^{*}\right)\right)^{\vartheta_{2}} .
\end{aligned}
$$

By triangle inequality and (47), for each $m$, we obtain

$$
\begin{aligned}
d_{L}\left(l^{*}, N\left(l^{*}, l^{*}, \ldots, l^{*}\right)\right) & \leq d_{L}\left(l^{*}, N\left(l_{m}, l_{m+1}, \ldots, l_{m+k-1}\right)\right)+d_{L}\left(N\left(l_{m}, l_{m+1}, \ldots, l_{m+k-1}\right), N\left(l^{*}, l^{*}, \ldots, l^{*}\right)\right) \\
& \leq d_{L}\left(l^{*}, l_{m+k}\right)+\zeta d_{L}\left(l_{m+k-1}, l_{m+k}\right)^{\vartheta_{1}} d_{L}\left(l^{*}, N\left(l^{*}, l^{*}, \ldots, l^{*}\right)\right)^{\vartheta_{2}} .
\end{aligned}
$$

Letting $m \longrightarrow \infty$ in $(48)$, we get $d_{L}\left(l^{*}, N\left(l^{*}, l^{*}, \ldots, l^{*}\right)\right)$ $=0$. Hence, the claim is true, that is, $l^{*}=N\left(l^{*}, l^{*}\right.$, $\left.\ldots, l^{*}\right)$.
Theorem 7. Consider an extended interpolative Prešić typeII contraction map $N: L^{k} \longrightarrow L$, for any fixed $k \in \mathbb{N}$, on a complete metric space $\left(L, d_{L}\right)$, that is, for each $s_{1}, s_{2}, \ldots, s_{k}$, 
$w_{1}, w_{2}, \ldots, w_{k}, \in L \backslash F i x(N) \quad$ with $\quad \min \left\{\gamma\left(s_{1}, w_{1}\right)\right.$, $\left.\gamma\left(s_{2}, w_{2}\right), \ldots, \gamma\left(s_{k}, w_{k}\right)\right\} \geq 1$, we get

$$
d_{L}\left(N\left(s_{1}, s_{2}, \ldots, s_{k}\right), N\left(w_{1}, w_{2}, \ldots, w_{k}\right)\right) \leq \zeta d_{L}\left(s_{k}, N\left(s_{1}, s_{2}, \ldots, s_{k}\right)\right)^{\vartheta_{1}} d_{L}\left(w_{k}, N\left(w_{1}, w_{2}, \ldots, w_{k}\right)\right)^{\vartheta_{2}}
$$

where $\gamma: L \times L \longrightarrow \mathbb{R}$ is a map, $\vartheta_{1}, \vartheta_{2} \in(0,1)$ with $\vartheta_{1}+\vartheta_{2}=1, \quad \zeta \in[0,1), \quad$ and $\operatorname{Fix}(N)=\{l \in L: l=N(l, l, \ldots, l)\}$. Also, consider that

(i) If $\min \left\{\gamma\left(s_{1}, w_{1}\right), \gamma\left(s_{2}, w_{2}\right), \ldots, \gamma\left(s_{k}, w_{k}\right)\right\} \geq 1$, then $\gamma\left(N\left(s_{1}, s_{2}, \ldots, s_{k}\right), N\left(w_{1}, w_{2}, \ldots, w_{k}\right)\right) \geq 1$.

(ii) There exist elements $s_{1}, s_{2}, \ldots, s_{k} \in L$, such that $\min \left\{\gamma\left(s_{1}, s_{2}\right), \gamma\left(s_{2}, s_{3}\right), \ldots, \gamma\left(s_{k}, N\left(s_{1}, s_{2}, \ldots, s_{k}\right)\right)\right\} \geq 1$.

(iii) For every sequence $\left\{l_{m}\right\}$ in $L$ with $\gamma\left(l_{m}, l_{m+1}\right) \geq 1 \forall m \geq m_{0}$ for some natural number $m_{0}$ and $l_{m} \longrightarrow l$, we have $\gamma\left(l_{m}, l\right) \geq 1 \forall m \geq m_{0}$.
Then, there exists at least one point of $L$ that satisfies the equation $l=N(\underbrace{l, l, \ldots, l}_{k \text {-times }})$.

Proof. The proof can be obtained on the same steps as the proofs of Theorems 6 and 2 are done.

The following theorems can be considered as an extended form of Theorems 3 and 5, respectively.

Theorem 8. Consider an extended interpolative Prešić type-I proximal contraction map $N: O_{L}^{k} \longrightarrow M_{L}$, for any fixed $k \in \mathbb{N}$, on a metric space $\left(L, d_{L}\right)$, that is, for each $s_{1}, s_{2}, \ldots, s_{k}$, $w_{1}, w_{2}, \ldots, w_{k}, p, q \in O_{L} \backslash \operatorname{Bes}(N)$ with

$$
d_{L}\left(p, N\left(s_{1}, s_{2}, \ldots, s_{k}\right)\right)=D_{L}\left(O_{L}, M_{L}\right)=d_{L}\left(q, N\left(w_{1}, w_{2}, \ldots, w_{k}\right)\right)
$$

we get

$d_{L}(p, q)^{\min \left\{\gamma\left(s_{1}, w_{1}\right), \gamma\left(s_{2}, w_{2}\right), \ldots, \gamma\left(s_{k}, w_{k}\right)\right\}} \leq \zeta d_{L}\left(s_{k}, p\right)^{\vartheta_{1}} d_{L}\left(w_{k}, q\right)^{\vartheta_{2}}$,

where $\gamma: O_{L} \times O_{L} \longrightarrow \mathbb{R} \backslash\{0\}$ is a map, $\vartheta_{1}, \vartheta_{2} \in(0,1)$ with $\vartheta_{1}+\vartheta_{2}=1, \quad \zeta \in[0,1), \quad \operatorname{Bes}(N)=\left\{o \in O_{L}: d_{L}(o, N(o, o\right.$, $\left.\ldots, o))=D_{L}\left(O_{L}, M_{L}\right)\right\}$, and $O_{L}, M_{L}$ are the nonvoid subsets of $L$. Also, consider that

(i) If $\min \left\{\gamma\left(s_{1}, w_{1}\right), \gamma\left(s_{2}, w_{2}\right), \ldots, \gamma\left(s_{k}, w_{k}\right)\right\}=1$ and $d_{L}\left(p, N\left(s_{1}, s_{2}, \ldots, s_{k}\right)\right)=D_{L} \quad\left(O_{L}, M_{L}\right)=d_{L}(q, N$ $\left.\left(w_{1}, w_{2}, \ldots, w_{k}\right)\right)$, then $\gamma(p, q)=1$.

(ii) There exist elements $s_{1}, s_{2}, \ldots, s_{k}, p \in O_{L}$ with $d_{L}\left(p, N\left(s_{1}, s_{2}, \ldots, s_{k}\right)\right)=D_{L}\left(O_{L}, M_{L}\right)$ and $\min \left\{\gamma\left(s_{1}, s_{2}\right), \gamma\left(s_{2}, s_{3}\right), \ldots, \gamma\left(s_{k}, p\right)\right\}=1$.

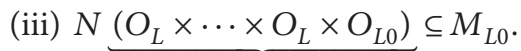

(iv) $O_{L_{0}}$ is nonempty and complete with respect to $d_{L}$.

(v) For every sequence $\left\{l_{m}\right\}$ in $O_{L_{0}}$ with $\gamma\left(l_{m}, l_{m+1}\right)=$ $1 \forall m \geq m_{0}$ for some natural number $m_{0}$ and $l_{m} \longrightarrow l$, we have $\gamma\left(l_{m}, l\right)=1 \forall m \geq m_{0}$.
Then, there exists at least one point of $L$ that satisfies the equation $d_{L}(l, N(\underbrace{l, l, \ldots, l}_{k \text {-times }}))=D_{L}\left(O_{L}, M_{L}\right)$.

Proof. By hypothesis (ii), we get $l_{1}, l_{2}, \ldots, l_{k}, l_{k+1}$ in $O_{L}$ with $d_{L}\left(l_{k+1}, N\left(l_{1}, l_{2}, \ldots, l_{k}\right)\right)=D_{L}\left(O_{L}, M_{L}\right)$ and

$$
\min \left\{\gamma\left(l_{1}, l_{2}\right), \gamma\left(l_{2}, l_{3}\right), \ldots, \gamma\left(l_{k}, l_{k+1}\right)\right\}=1 .
$$

Hypothesis (iii) implies that $N\left(l_{2}, l_{3}, \ldots, l_{k+1}\right) \in M_{L 0}$, and there is $l_{k+2} \in O_{L_{0}}$ satisfying

$$
d_{L}\left(l_{k+2}, N\left(l_{2}, l_{3}, \ldots, l_{k+1}\right)\right)=D_{L}\left(O_{L}, M_{L}\right) .
$$

Then, from hypothesis (i), we get $\gamma\left(l_{k+1}, l_{k+2}\right)=1$. Repeated use of hypotheses (i), (ii), and (iii) yields $\gamma\left(l_{m}, l_{m+1}\right)=$ 1 and $d_{L}\left(l_{m+k}, N\left(l_{m}, l_{m+1}, \ldots, l_{m+k-1}\right)\right)=D_{L}\left(O_{L}, M_{L}\right)$ for all $m \in \mathbb{N}$. As

$$
d_{L}\left(l_{m+k}, N\left(l_{m}, l_{m+1}, \ldots, l_{m+k-1}\right)\right)=D_{L}\left(O_{L}, M_{L}\right),
$$

and

$$
d_{L}\left(l_{m+k+1}, N\left(l_{m+2}, l_{m+2}, \ldots, l_{m+k}\right)\right)=D_{L}\left(O_{L}, M_{L}\right), \quad \forall m \in \mathbb{N},
$$

and

$$
\min \left\{\gamma\left(l_{m}, l_{m+1}\right), \gamma\left(l_{m+1}, l_{m+2}\right), \ldots, \gamma\left(l_{m+k-1}, l_{m+k}\right)\right\}=1, \quad \forall m \in \mathbb{N}
$$

Then, by (52), we get 


$$
\begin{aligned}
d_{L}\left(l_{m+k}, l_{m+k+1}\right) & =d_{L}\left(l_{m+k}, l_{m+k+1}\right)^{\min \left\{\gamma\left(l_{m}, l_{m+1}\right), \ldots, \gamma\left(l_{m+k-1}, l_{m+k}\right)\right\}}, \\
& \leq \zeta d_{L}\left(l_{m+k-1}, l_{m+k}\right)^{\vartheta_{1}} d_{L}\left(l_{m+k}, l_{m+k+1}\right)^{\vartheta_{2}}, \quad \forall m \in \mathbb{N} .
\end{aligned}
$$

Following the proof of Theorem 6, we say that $\left\{l_{m}\right\}_{m \geq k+1}$ is Cauchy in $O_{L 0}$, and by the completeness of $O_{L 0}$, we get a point $l^{*} \in O_{L 0}$ with $l_{m} \longrightarrow l^{*}$. Also, by (v), we get $\gamma\left(l_{m}, l^{*}\right)=1 \forall m \in \mathbb{N}$, since $\gamma\left(l_{m}, l_{m+1}\right)=1$ and $l_{m} \longrightarrow l^{*}$. Clearly, $N\left(l^{*}, l^{*}, \ldots, l^{*}\right) \in M_{L 0}$, and there is $w^{*} \in O_{L 0}$ with $d_{L}\left(w^{*}, N\left(l^{*}, l^{*}, \ldots, l^{*}\right)\right)=D_{L}\left(O_{L}, M_{L}\right)$. Here, the claim is $w^{*}=l^{*}$. Suppose it is wrong, then by (52), for each $m \in \mathbb{N}$, we get

$$
\begin{aligned}
& d_{L}\left(l_{m+k}, w^{*}\right)=d_{L}\left(l_{m+1}, w^{*}\right)^{\min \left\{\gamma\left(l_{m}, l^{*}\right), \gamma\left(l_{m+1}, l^{*}\right), \ldots, \gamma\left(l_{m+k-1}, l^{*}\right)\right\}} \\
& \leq \zeta d_{L}\left(l_{m+k-1}, l_{m+k}\right)^{\vartheta_{1}} d_{L}\left(l^{*}, w^{*}\right)^{\vartheta_{2}} .
\end{aligned}
$$

Letting $m \longrightarrow \infty$ in $(60)$, we obtain $d_{L}\left(l^{*}, w^{*}\right)=0$, and it contradicts our assumption. Hence, our claim is true, that is, $\quad l^{*}=w^{*}$. Therefore, $\quad d_{L}\left(l^{*}, N\left(l^{*}, l^{*}, \ldots, l^{*}\right)\right)=$ $D_{L}\left(O_{L}, M_{L}\right)$.

Theorem 9. Consider an extended interpolative Prešić typeII proximal contraction map $N: O_{L}^{k} \longrightarrow M_{L}$, for any fixed $k \in \mathbb{N}$, on a metric space $\left(L, d_{L}\right)$, that is, for each $s_{1}, s_{2}, \ldots, s_{k}$, $w_{1}, w_{2}, \ldots, w_{k}, p, q \in O_{L} \backslash \operatorname{Bes}(N)$ with

$$
d_{L}\left(p, N\left(s_{1}, s_{2}, \ldots, s_{k}\right)\right)=D_{L}\left(O_{L}, M_{L}\right)=d_{L}\left(q, N\left(w_{1}, w_{2}, \ldots, w_{k}\right)\right)
$$

and

$$
\min \left\{\gamma\left(s_{1}, w_{1}\right), \gamma\left(s_{2}, w_{2}\right), \ldots, \gamma\left(s_{k}, w_{k}\right)\right\} \geq 1,
$$

we get

$$
d_{L}(p, q) \leq \zeta d_{L}\left(s_{k}, p\right)^{\vartheta_{1}} d_{L}\left(w_{k}, q\right)^{\vartheta_{2}}
$$

where $\gamma: O_{L} \times O_{L} \longrightarrow \mathbb{R}$ is a map, $\vartheta_{1}, \vartheta_{2} \in(0,1)$ with $\vartheta_{1}+\vartheta_{2}=1$, and $\zeta \in[0,1)$. Also, consider that

(i) If $\min \left\{\gamma\left(s_{1}, w_{1}\right), \gamma\left(s_{2}, w_{2}\right), \ldots, \gamma\left(s_{k}, w_{k}\right)\right\} \geq 1$ and $d_{L}\left(p, N\left(s_{1}, s_{2}, \ldots, s_{k}\right)\right)=D_{L}\left(O_{L}, M_{L}\right)=d_{L}(q, N$ $\left.\left(w_{1}, w_{2}, \ldots, w_{k}\right)\right)$, then $\gamma(p, q) \geq 1$.

(ii) There exist elements $s_{1}, s_{2}, \ldots, s_{k}, p \in O_{L}$ with $d_{L}\left(p, N\left(s_{1}, s_{2}, \ldots, s_{k}\right)\right)=D_{L}\left(O_{L}, M_{L}\right)$ and

$\min \left\{\gamma\left(s_{1}, s_{2}\right), \gamma\left(s_{2}, s_{3}\right), \ldots, \gamma\left(s_{k}, p\right)\right\} \geq 1$.

(iii) $N \underbrace{\left(O_{L} \times \cdots \times O_{L} \times O_{L 0}\right)}_{k-1 \text { times }} \subseteq M_{L 0}$.

(iv) $O_{L_{0}}$ is nonempty and complete with respect to $d_{L}$.

(v) For every sequence $\left\{l_{m}\right\}$ in $O_{L_{0}}$ with $\gamma\left(l_{m}, l_{m+1}\right) \geq 1 \forall m \geq m_{0}$ for some natural number $m_{0}$ and $l_{m} \longrightarrow l$, we have $\gamma\left(l_{m}, l\right) \geq 1 \forall m \geq m_{0}$.

Then, there exists at least one point of $L$ that satisfies the equation $d_{L}(l, N(\underbrace{l, l, \ldots, l}_{k \text {-times }}))=D_{L}\left(O_{L}, M_{L}\right)$.

The proof of the above theorem can be derived by viewing the proof of Theorem 8 .

\section{Conclusion}

This article provides a few results dealing with fixed points and best proximity points of the mappings defined on product spaces. The notions of interpolative Prešić type contractions and interpolative Prešić type proximal contractions are introduced in the context of metric spaces to discuss the existence of fixed points and best proximity points of such maps, respectively. These notions are derived by considering the concept of interpolative Kannan contraction.

\section{Data Availability}

The data used to support the findings of this study are available from the corresponding author upon request.

\section{Conflicts of Interest}

The authors declare that they have no conflicts of interest.

\section{Authors' Contributions}

All authors contributed equally in this article and approved the final manuscript.

\section{Acknowledgments}

This work was supported by the Deanship of Scientific Research (DSR), King Abdulaziz University, Jeddah, Saudi Arabia, under grant number KEP-5-130-42. The authors, therefore, gratefully acknowledge the DSR technical and financial support.

\section{References}

[1] S. B. Prešić, "Sur la convergence des suites," Comptes rendus hebdomadaires des séances de l'Académie des sciences, vol. 260, no. 14, pp. 3828-3830, 1965.

[2] L. B. Ćirić and S. B. Prešić, "On Prešić type generalisation of Banach contraction principle," Acta Mathematica Universitatis Comenianae, vol. 76, no. 2, pp. 143-147, 2007. 
[3] M. S. Khan, M. Berzig, and B. Samet, "Some convergence results for iterative sequences of Prešić type and applications," Advances in Difference Equations, vol. 38, 2012.

[4] S. Shukla and S. Radenović, "Some generalizations of Prešić type mappings and applications," Annals of the Alexandru Ioan Cuza University - Mathematics, vol. 15, 2015.

[5] S. Shukla, R. Sen, and S. Radenović, "Set-valued Prešić type contraction in metric spaces," Annals of the Alexandru Ioan Cuza University - Mathematics, vol. 24, 2014.

[6] R. Kannan, "Some results on fixed point," Bulletin of the Calcutta Mathematical Society, vol. 60, pp. 71-76, 1968.

[7] S. K. Chatterjea, "Fixed point theorem," Comptes rendus de l'Academie bulgare des Sciences, vol. 25, pp. 727-730, 1972.

[8] E. Karapinar, "Revisiting the Kannan type contractions via interpolation," Advances in the Theory of Nonlinear Analysis and its Application, vol. 2, no. 2, pp. 85-87, 2018.

[9] E. Karapınar, R. P. Agarwal, and H. Aydi, "Interpolative Reich-Rus-Ćirić type contractions on partial metric spaces," Mathematics, vol. 6, no. 11, 2018.

[10] H. Aydi, C. M. Chen, and E. Karapınar, "Interpolative ĆirićReich-Rus type contractions via the Branciari distance," Mathematics, vol. 7, no. 1, 2019.

[11] B. Mohammadi, V. Parvaneh, and H. Aydi, "On extended interpolative Ćirić-Reich-Rus type F-contractions and an application," Journal of Inequalities and Applications, vol. 290, no. 1, 2019.

[12] E. Karapinar, O. Algahtani, and H. Aydi, "On interpolative Hardy-Rogers type contractions," Symmetry, vol. 11, no. 1, 2019.

[13] P. Debnath and M. de La Sen, "Set-valued interpolative Hardy-Rogers and set-valued Reich-Rus-Ćirić-type contractions in b-metric spaces," Mathematics, vol. 7, no. 9, 2019.

[14] M. Sarwar, W. Ahmad, and T. Abdeljawad, "Fixed point theorems for rational interpolative-type operators with applications," Journal of Function Spaces, vol. 2020, Article ID 7816505, , 2020.

[15] M. S. Khan and Y. M. Singh, "On the interpolative $(\phi, \psi)$ type Z- contraction," University Politehnica of Bucharest Scientific Bulletin-Series A-Applied Mathematics and Physics, vol. 83, no. 2, pp. 25-38, 2021.

[16] I. Altun and A. Tasdemir, "On best proximity points of interpolative proximal contractions," Quaestiones Mathematicae, vol. 44, no. 9, pp. 1233-1241, 2021.

[17] A. Fulga and S. S. Yesilkaya, "On some interpolative contractions of Suzuki type mappings," Journal of Function Spaces, vol. 2021, Article ID 6596096, , 2020.

[18] E. Karapinar, A. Fulga, and A. F. Roldán López de Hierro, "Fixed point theory in the setting of $(\alpha, \beta, \psi, \phi)$-interpolative contractions," Advances in Difference Equations, vol. 339, 2021.

[19] M. Alansari and M. U. Ali, "Unified multivalued interpolative Reich-Rus-Ćirić-type contractions," Advances in Difference Equations, vol. 311, 2021.

[20] Y. U. Gaba and E. Karapınar, "A new approach to the interpolative contractions," Axioms, vol. 8, no. 4, 2019.

[21] M. Alansari and M. U. Ali, "On interpolative F-contractions with shrink map," Advances in Difference Equations, vol. 282, 2021.

[22] J. Caballero, J. Harjani, and K. Sadarangani, "A Best proximity point theorem for geraghty-contractions," Fixed Point Theory and Applications, vol. 231, 2012.

[23] N. Bilgili, E. Karapınar, and K. Sadarangani, "A generalization for the best proximity point of Geraghty-contractions," Journal of Inequalities and Applications, vol. 286, 2013.
[24] H. Aydi, E. Karapınar, I. M. Erhan, and P. Salimi, "Best proximity points of generalized almost $\psi$-geraghty contractive non-self mappings," Fixed Point Theory and Applications, vol. 32, 2014.

[25] A. Pitea, "Best proximity results on dualistic partial metric spaces," Symmetry, vol. 11, no. 3, 2019.

[26] S. S. Basha and N. Shahzad, "Best proximity point theorems for generalized proximal contractions," Fixed Point Theory and Applications, vol. 42, 2012.

[27] S. S. Basha, "Best proximity point theorems for some special proximal contractions," Numerical Functional Analysis and Optimization, vol. 40, no. 10, pp. 1182-1193, 2019.

[28] M. Jleli and B. Samet, "Best proximity points for $\alpha-\psi$-proximal contractive type mappings and applications $\alpha-\psi$-proximal contraction type mappings and applications," Bulletin des Sciences Mathematiques, vol. 137, no. 8, pp. 977-995, 2013.

[29] M. Jleli, E. Karapınar, and B. Samet, "Best proximity points for generalized $\alpha-\psi$-proximal contractive type mappings," Journal of Applied Mathematics, vol. 2013, Article ID 534127, , 2013.

[30] H. Aydi, A. Felhi, and E. Karapınar, "On common best proximity points for generalized $\alpha$ - $\psi$-proximal contractions," The Journal of Nonlinear Science and Applications, vol. 9, no. 5, pp. 2658-2670, 2016.

[31] A. Abkar and M. Gabeleh, "The existence of best proximity points for multivalued non-self-mappings," Revista de la Real Academia de Ciencias Exactas, Fisicas y Naturales. Serie A. Matematicas, vol. 107, no. 2, pp. 319-325, 2013.

[32] P. Kumam, H. Aydi, E. Karapınar, and W. Sintunavarat, "Best proximity points and extension of mizoguchi-takahashi's fixed point theorems," Fixed Point Theory and Applications, vol. 242, 2013.

[33] M. U. Ali, H. Houmani, and T. Kamran, "New type of proximal contractions via implicit simulation functions," Journal of Nonlinear Convex and Analysis, vol. 20, no. 3, pp. 435-445, 2019.

[34] H. Sahin, M. Aslantas, and I. Altun, "Best proximity and best periodic points for proximal nonunique contractions," Journal of Fixed Point Theory and Applications, vol. 23, no. 4, pp. 1-14, 2021.

[35] M. U. Ali, M. Farheen, T. Kamran, and G. Maniu, "Prešić type non-self operators and related best proximity results," Mathematics, vol. 7, no. 5, 2019. 